\title{
Pleural fluid penetration of moxifloxacin and doripenem: An experimental model of empyema
}

\author{
(1) Mustafa Calik, ${ }^{1}$ (D) Saniye Goknil Calik, ${ }^{2}$ () Mustafa Dagli, ${ }^{3}$ () Recep Kesli, ${ }^{4}$ () Hidir Esme ${ }^{1}$ \\ ${ }^{1}$ Department of Thoracic Surgery, Health Sciences University, Konya Training and Research Hospital, Konya, Turkey \\ Emergency and First Aid Program, Vocational School of Health Services KTO Karatay University, Konya, Turkey \\ ${ }^{3}$ Department of Cardiovascular Surgery, Health Sciences University, Konya Training and Research Hospital, Konya, Turkey \\ ${ }^{4}$ Department of Microbiology, Health Sciences University, Konya Training and Research Hospital, Konya, Turkey
}

\begin{abstract}
OBJECTIVE: This study aimed to evaluate the penetration of moxifloxacin and doripenem into the pleural fluid (PF) using a rabbit model of empyema.

METHODS: An empyema was induced using the intrapleural injection of turpentine $(1 \mathrm{~mL})$, followed $24 \mathrm{~h}$ later by instillation of $5 \mathrm{~mL}$ Klebsiella Pneumoniae (ATCC 33495), Fusobacterium nucleatum (ATCC 25586) and Streptokok Pneumoniae (ATCC $6305)$ into the pleural space. After an empyema was corroborated, Moxifloxacin $\left(25 \mathrm{mg} / \mathrm{kg}^{-1}\right)$ and Doripenem $\left(20 \mathrm{mg} / \mathrm{kg}^{-1}\right)$ were administered intraperitoneally. To determine the levels of antibiotics measured by High-Performance Liquid Chromatography in pleural and blood samples were obtained serially at $8,24,48$ and $72^{\text {nd }}$ hour.

RESULTS: The penetration of both antibiotics into the PF was very good. The penetration rate of doripenem (area under the curve (AUC) for PF/blood (AUCPF/AUCblood) ratio=1.68) was better than moxifloxacin (ratio=0.78). Equalization time between the PF and blood concentration of doripenem was more quickly than moxifloxacin. Peak PF concentration of moxifloxacin was $0,81 \mu \mathrm{g} / \mathrm{mL}^{-1}$ and occurred $8 \mathrm{~h}$ after infusion and then gradually decreased; at the beginning of the blood and pleural fluid concentrations of doripenem were equal. While the pleura concentration was increasing, blood concentration was almost the same. Doripenem reached a peak concentration $(0.54 \mu \mathrm{g} / \mathrm{ml}) 24 \mathrm{~h}$ post-administration.
\end{abstract}

CONCLUSION: Differences were found in the penetration of the two antibiotics. Doripenem had convenient penetration PF compared to moxifloxacin. Due to the differences between human and rabbit pleural thickness, doripenem's pleural penetration should be examined in infection models in animals with equal pleura thickness and clinical trials.

Keywords: Chromatography; doripenem; empyema; moxifloxacin; turpentine.

Cite this article as: Calik M, Goknil Calik S, Dagli M, Kesli R, Esme H. Pleural fluid penetration of moxifloxacin and doripenem: An experimental model of empyema. North Clin Istanb 2020;7(2):99-105. Empyema is defined as pus that contains leukocytes;
bacteria and cellular debris in the pleural space depend on the miscellaneous etiology, localized or involving the entire pleural cavity [1]. Although empyema was first described by the Egyptian physician Imhotep 3000 years ago Hippocrates, who defines him than 2.500 years later, around $500 \mathrm{BC}$ was more famous [2]; since that time, it continues to remain a significant medical problem [3]. Post-parapneumonic effusions (40-60\%) and postsurgical empyema $(30 \%)$ that defined as the development of infection in the pleural cavity after thoracic surgery are the most common causes of empyema [1]. The most common pathogens isolated in pleural empyema are both aerobic and anaerobic bacteria. Streptococci (Strep-

Received: March 18, 2018 Accepted: May 19, 2019 Online: July 02, 2019

Correspondence: Dr. Mustafa CALIK. Saglik Bilimleri Universitesi, Konya Egitim ve Arastirma Hastanesi, Gogus Cerrahisi Klinigi, Meram Yerleskesi, Haci Saban Mahhallesi, Meram Yeniyol Caddesi, No: 97, 42090 Meram, Konya, Turkey.

Tel: +903323236709 e-mail: drmcalik@hotmail.com

(c) Copyright 2020 by Istanbul Provincial Directorate of Health - Available online at www.northclinist.com 
tococcus pneumonia) and staphylococci (Staphylococcus aureus) dominate gram-positive isolates, while Klebsiella spp., Pseudomonas spp. Moreover, Haemophilus influenza is the most common gram-negative isolates. Anaerobic organisms are often found in combination with other microorganisms [4].

The treatment generally is determined by the symptoms and severity or phase of the disease. Even though there have been several treatment options, conventional initial treatment is chest tube placement to evacuating pleural fluid in enabling lung re-expansion and ameliorate lung function with empiric antibiotic therapy for the causative organism [5]. Bacterial pneumonia has an associated parapneumonic pleural effusion that evolved less than $10 \%$ from complicated parapneumonic effusions to empyema in approximately $40 \%$ of cases. Mixed infections accompanied by gram-positive and gram-negative, as well as anaerobic bacteria, are observed in many patients with empyema. However, many antibiotics, including second and third-generation cephalosporins, $\beta$ lactam- $\beta$-lactamase inhibitor combinations, macrolides, fluoroquinolones, metronidazole, clindamycin, carbapenems, aminoglycosides or aztreonam, are used to treat in these circumstances [6]. Antibiotics, such as carbapenems and fluoroquinolones, are administered frequently [7]. Fluoroquinolones, such as moxifloxacin, which are derivatives of nalidixic acid, exhibit favorable activity against both Gram-positive and Gram-negative bacilli. Fluoroquinolones offer higher efficacy with highly resistant pneumococci, and/or higher anaerobic coverage, providing advantages for treating patients with empyema [6]. Doripenem, a member of the $\beta$-lactam class of antibiotics, is one of the newest addition to the carbapenems. Doripenem exhibits concentration-independent bactericidal activity against gram-positive bacteria; enteric and non-enteric gram-negative bacteria, including extendedspectrum $\beta$-lactamase-producing strains; and anaerobic pathogens [8]. This study aimed to evaluate the penetration of moxifloxacin and doripenem into the blood and pleural fluid $(\mathrm{PF})$ using a rabbit model of empyema after single intraperitoneal administration.

\section{MATERIALS AND METHODS}

\section{Animals}

A total of 30 white New Zealand male rabbits (weight range 2,1-3,3 kg) were used for this study. All animals received humane care and were used in compliance with standards established by the European Convention for
Animal Care and Use of Laboratory Animals. The rabbits were fed with a standard pelleted diet and were allowed to access tap water ad libitum. The animals were housed in standard individual cages on a 12 -hour $\mathrm{h}$ light/dark cycle at room temperature in a humidity-controlled environment. The local Animal Ethical Committee of this study approved all study-related procedures. This study was approved and funded by the School of Medicine Animal Care and Investigational Committee at our institution, which we gratefully acknowledge.

\section{Bacteria Preparation}

The Streptococci Pneumoniae (ATCC 6305) and Klebsiella Pneumoniae (ATCC 33495) strain were grown respectively on blood agar and McConkey agar (Becton Dickinson, Sparks, MD, USA) for $24 \mathrm{~h}$ at 35 OC. Fusobacterium nucleatum (ATCC 25586) strain was grown on Schaedler Broth for $48 \mathrm{~h}$ at $350 \mathrm{C}$. After it was grown, $1 \times 1010$ bacteria (in a $5-\mathrm{mL}$ volume of saline solution) were injected into the right pleural space.

\section{Empyema Induction}

General anesthesia was induced using Ketamine $\mathrm{HCl}$ (Ketanest, Pfizer Pharma GmbH, Karlsruhe, Germany) $15--20 \mathrm{mg} / \mathrm{kg}$ i.v* or $20--25 \mathrm{mg} / \mathrm{kg}$ i.m. and maintained with Xylazine (Alfazyne 2\%; Alfasan International. BV, Woerden, Netherlands) $0.5--1 \mathrm{mg} / \mathrm{kg}$ i.v. or $1--2 \mathrm{mg} /$ $\mathrm{kg}$ i.m. If needed, same doses of Ketamine $\mathrm{HCl}$ and Xylazine were repeated with the emergence of reflex responses (pedal reflex, palpebral and corneal reflexes) to keep constant anesthesia. Body temperature was monitored by inserting a heat probe into the ECG and rectum by the aid of needle electrodes. Heating lamps were used to keep the animals at $37^{\circ} \mathrm{C} \pm 5^{\circ} \mathrm{C}$ body temperature during the surgical preparation and working periods. The mean anesthesia time was $5-10$ min for each rabbit. All subjects were placed in a lateral decubitus position on an operating table. The right chest wall of each rabbit was shaved and then scrubbed with povidone-iodine (10\% povidone-iodine, Betadine, Kansuk, Istanbul, Turkey). An 18-gauge catheter was transfixed over the $5^{\text {th }}$ intercostal space into the right thoracic cavity using the modified Seldinger technique. An 18-gauge catheter was attached to a Heimlich valve with a three-way stopcock in-line between the catheter and the Heimlich valve. After its placement, any air within the pleural space was aspirated via the catheter. $1 \mathrm{~mL}$ of Turpentine oil (SigmaAldrich $\mathrm{GMBH}$, Germany) was administered into the 
pleural space through the catheter, and then flushed with $1.5 \mathrm{~mL}$ of saline solution.

An empyema was induced using the intrapleural injection of turpentine $(1 \mathrm{~mL})$, followed $24 \mathrm{~h}$ later by instillation of Klebsiella Pneumoniae (ATCC 33495) and Fusobacterium nucleatum (ATCC 25586) (1x1010 cfu in a final volume of $5 \mathrm{~mL}$ saline) in doripenem group and Streptokok Pneumoniae (ATCC 6305) (1x1010 cfu in a final volume of $5 \mathrm{~mL}$ saline) in moxifloxacin group into the pleural space.

\section{Empyema Verification}

A maximum of $1 \mathrm{~mL}$ of pleural fluid was removed for anal$y$ sis at $8,24,48$ and $72 \mathrm{~h}$ after bacterial injection. The $\mathrm{pH}$ and the glucose of the pleural fluid were analyzed using a blood gas machine (ABL800 FLEX Blood Gas Analyzer Radiometer Medical ApS, Denmark), and lactate-D-hydrogenase $(\mathrm{LDH})$ levels were analyzed using a standard biochemistry laboratory (AU5800 Clinical Chemistry System Beckman Coulter, Inc, CA; US). An empyema was said to be present if the pleural fluid appeared grossly infected, if the glucose was $<40 \mathrm{mg} / \mathrm{dL}^{-1}$ and if the pleural fluid $\mathrm{pH}$ was $<7.10$ and the $\mathrm{LDH}$ was $>1.000 \mathrm{U} / \mathrm{L}^{-1}$.

\section{Antibiotic Administration}

After an empyema was corroborated, Moxifloxacin (25 mg/ $\mathrm{kg}^{-1}$ ) (Avelox, Bayer, Istanbul, Turkey) and Doripenem $\left(20 \mathrm{mg} / \mathrm{kg}^{-1}\right)$ (Janssen-Cilag, NJ, USA) were administered intraperitoneally over three minutes.

\section{Pleural Fluid and Blood Specimens}

Immediately after the specimens were collected, the blood and the pleural fluid samples were centrifuged at $3,000 \mathrm{rpm}$ for $15 \mathrm{~min}$. The supernatants were then re- frigerated at $-80^{\circ} \mathrm{C}$ overnight. To determine the levels of antibiotics measured by High-Performance Liquid Chromatography in pleural and blood samples were obtained serially at $8,24,48$ and $72^{\text {nd }}$ hour.

\section{Postoperative Care and Follow-up}

The animals were followed-up for about three days after empyema verification. All animals were euthanized using a lethal IV dose of Nonbarbiturate anesthetic ( $\mathrm{Ke}$ tamine/Xylazine) painlessly according to the existing instructions established by the latest report of the AVMA Panel on Euthanasia. Three times the anesthetic dose was used for euthanasia [9].

\section{Statistical Analysis}

All data were entered into Microsoft Office Excel 2010 for Windows (Microsoft Corp., Redmond, WA, USA) and transferred into Statistical Package for Social Sciences version 18 (SPSS v18) (IBM Corp., Chicago, IL, USA) package software was used for data analysis. The relationships between the pleural fluid and the serum antibiotic levels and p-value are shown in Table 1 and 2. These values were analyzed primarily. The variables were presented as median $(25 \%-75 \%)$. The changes of variables in time were assessed using the Friedman test. When a significant difference was determined, then, pairwise comparisons were performed with the Wilcoxon signed-rank test with Bonferroni correction. The areas under the time-concentration curves (AUC) were estimated by the trapezoidal rule.

\section{RESULTS}

The median pleural doripenem concentrations were significantly different from each other at different sampling

TABLE 1. Levels of the antibiotics in the pleural fluid after i.p. administration

\begin{tabular}{|c|c|c|c|c|c|c|c|c|c|c|c|}
\hline \multirow[b]{2}{*}{ Pleural } & \multirow[b]{2}{*}{ Hour 8} & \multirow[b]{2}{*}{ Hour 24} & \multirow[b]{2}{*}{ Hour 48} & \multirow[b]{2}{*}{ Hour 72} & \multirow[b]{2}{*}{$\mathrm{p}^{*}$} & \multicolumn{6}{|c|}{ p-values of paired comparisons } \\
\hline & & & & & & I-II & I-III & I-IV & II-III & II-IV & III-IV \\
\hline Doripenem & $\begin{array}{c}0.15 \\
(0.12-0.30)\end{array}$ & $\begin{array}{c}0.30 \\
(0.17-0.51)\end{array}$ & $\begin{array}{c}0.63 \\
(0.27-0.89)\end{array}$ & $\begin{array}{c}0.33 \\
(0.19-0.49)\end{array}$ & 0.032 & 0.115 & 0.008 & 0.083 & 0.054 & 0.480 & 0.024 \\
\hline Moxifloxacin & $\begin{array}{c}0.65 \\
(0.35-1.43)\end{array}$ & $\begin{array}{c}0.26 \\
(0.24-0.31)\end{array}$ & $\begin{array}{c}0.18 \\
(0.17-0.24)\end{array}$ & $\begin{array}{c}0.03 \\
(0.03-0.04)\end{array}$ & 0.001 & 0.028 & 0.028 & 0.005 & 0.039 & 0.018 & 0.026 \\
\hline
\end{tabular}

*P-values for the changes of the variables in time. 
TABLE 2. Levels of the antibiotics in the blood after i.p. administration

\begin{tabular}{|c|c|c|c|c|c|c|c|c|c|c|c|}
\hline \multirow[b]{2}{*}{ Blood } & \multirow[b]{2}{*}{ Hour 8} & \multirow[b]{2}{*}{ Hour 24} & \multirow[b]{2}{*}{ Hour 48} & \multirow[b]{2}{*}{ Hour 72} & \multirow[b]{2}{*}{$\mathrm{p}^{*}$} & \multicolumn{6}{|c|}{ p-values of paired comparisons } \\
\hline & & & & & & I-II & I-III & I-IV & II-III & II-IV & III-IV \\
\hline Doripenem & $\begin{array}{c}0.30 \\
(0.14-0.36)\end{array}$ & $\begin{array}{c}0.21 \\
(0.17-0.32)\end{array}$ & $\begin{array}{c}0.21 \\
(0.18-0.28)\end{array}$ & $\begin{array}{c}0.21 \\
(0.18-0.31)\end{array}$ & 0.796 & & & & & & \\
\hline Moxifloxacin & $\begin{array}{c}1.23 \\
(1.01-1.35)\end{array}$ & $\begin{array}{c}0.39 \\
(0.18-0.52)\end{array}$ & $\begin{array}{c}0.17 \\
(0.11-0.25)\end{array}$ & $\begin{array}{c}0.14 \\
(0.08-0.31)\end{array}$ & 0.014 & 0.046 & 0.028 & 0.027 & 0.015 & 0.207 & 0.623 \\
\hline
\end{tabular}

$*$ P-values for the changes of the variables in time.

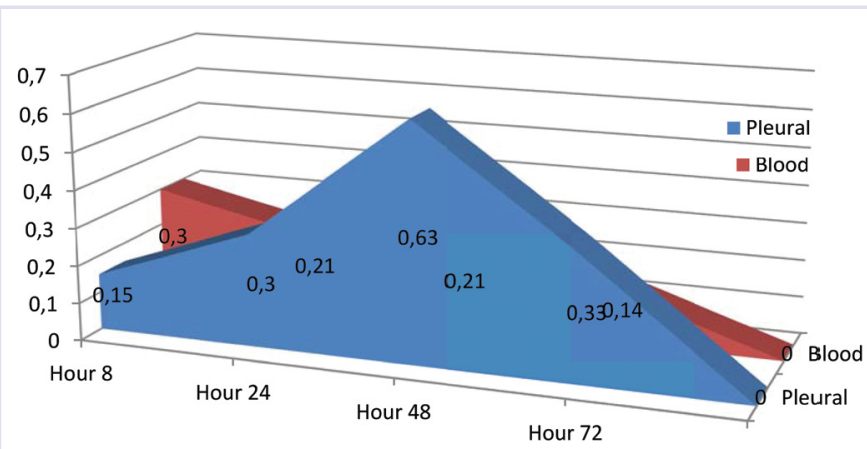

FIGURE 1. Median pleural and blood doripenem concentrations at hours $8,24,48$, and 72 .

times $(\mathrm{p}=0.032)$. The highest pleural doripenem concentration was achieved at hour $48(\mathrm{p}=0.008$ for hour $8-48$, $\mathrm{p}=0.054$ for hour $24-48, \mathrm{p}=0.024$ for hour $48-72$ ) (Fig. $1)$. The highest pleural moxifloxacin concentration was achieved at hour 8 . The decrease in median pleural moxifloxacin concentrations at hours 24,48 , and 72 was statistically significant $(\mathrm{p}=0.028$ for hour $8-24, \mathrm{p}=0.039$ for hour 24-48, $p=0.026$ for hour 48-72) (Fig. 2). The median pleural doripenem and moxifloxacin concentrations were significantly different from each other at hours 8,48 , and $72(\mathrm{p}=0.001$ for an hour $8, \mathrm{p}=0.002$ for hour 48, $<<0.001$ for hour 72 ). There was no significant change by time concerning the median blood doripenem concentrations $(p=0.796)$ (Table 1$)$. The highest blood moxifloxacin concentration was achieved at hour 8 . The decrease in median blood moxifloxacin concentrations at hours 24 and 48 was statistically significant $(\mathrm{p}=0.046$ for hour 8-24, $\mathrm{p}=0.015$ for hour 24-48). The median blood doripenem and moxifloxacin concentrations were significantly different from each other at hours 8,48 , and $72(p=0.001$ for an hour $8, p=0.016$ for hour 48 , $p=0.019$ for hour 72 ) (Table 2 ). The penetration of both

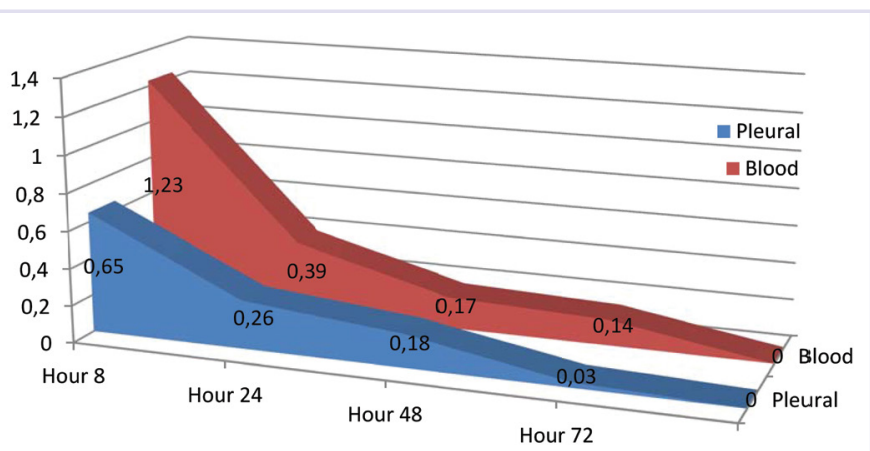

FIGURE 2. Median pleural and blood moxifloxacin concentrations at hours $8,24,48$, and 72 .

antibiotics into the PF was excellent. The penetration rate of doripenem (area under the curve (AUC) for PF/ blood (AUCPF/AUC blood) ratio=1.68) was better than moxifloxacin (ratio=0.78) (Fig. 3A, B). Equalization time between the PF and blood concentration of doripenem was more quickly than moxifloxacin. Peak PF concentration of moxifloxacin was $0.81 \mu \mathrm{g} / \mathrm{mL}^{-1}$ and occurred eight hours after infusion and then gradually decreased. At the beginning of the blood and pleural fluid concentrations of doripenem were equal. While the pleura concentration was increasing, blood concentration was almost the same. Doripenem reached a peak concentration $(0.54 \mu \mathrm{g} / \mathrm{ml}) 24 \mathrm{~h}$ post-administration.

\section{DISCUSSION}

The pleural cavity infections have been known for nearly 5000 years. Until now, there has been steadily in progress in microbiology, antibiotics, operations and other areas, but still seriously affect human health [10]. All over the world, the incidence of pleural cavity infection has been continuously increasing for all age groups. In only 12 years 

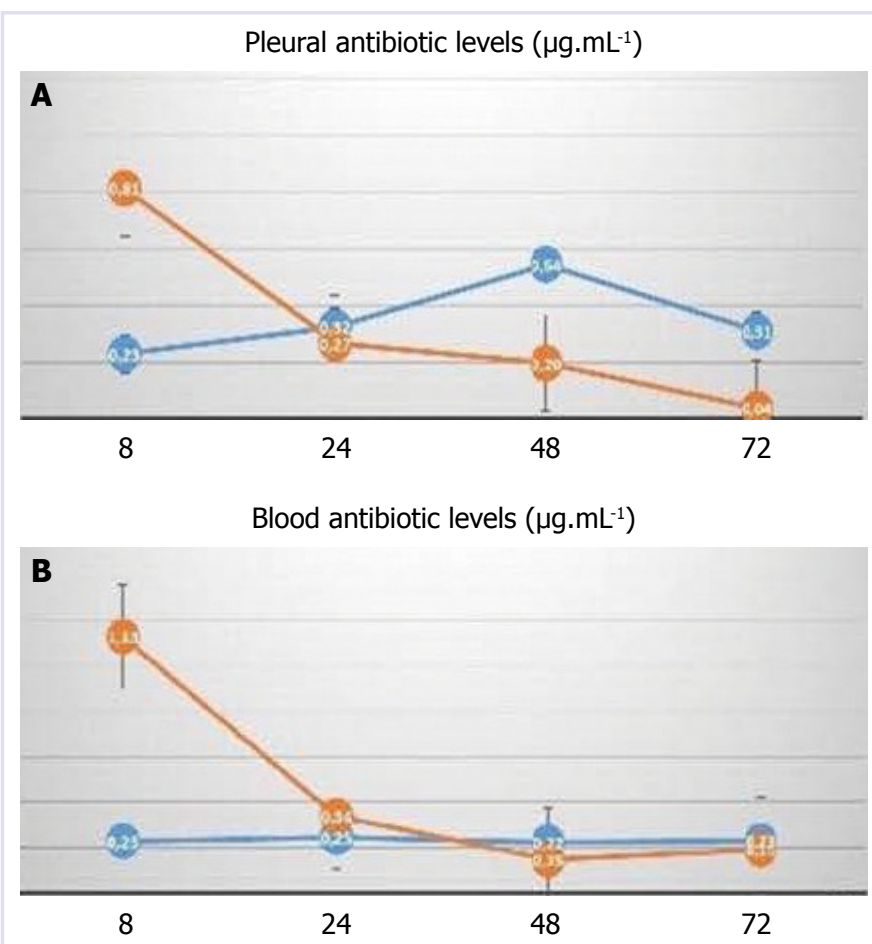

FIGURE 3. (A) Pleural antibiotic levels $\left(\mu \mathrm{g} \cdot \mathrm{mL}^{-1}\right)$ (B) Pleural antibiotic levels $\left(\mu \mathrm{g} \cdot \mathrm{mL}^{-1}\right)$.

from 1996 to 2008, the admission rate for empyema patients in the United States doubled from 3.04/100.000 to $5.98 / 100.000$. Thus, each year, about 80.000 British and American adults suffered from a pleural cavity infection that resulted in medical spending of approximately $\$ 500$ million. Also, the mortality rate is $20 \%$ even today. The mortality rate for older adults with the underlying diseases has reached up to $30 \%$. This may be related to weak underlying conditions of patients, inappropriate antibiotic selection or antimicrobial resistance and other factors $[10$, 11]. These changes are likely to increase the awareness of clinical diagnosis and the number of better diagnostic methods. The microbiological features of pleural infection have altered significantly in modern times, particularly since the introduction of antibiotic therapies in the 1940s. Microbiological pathogens that cause empyema vary according to etiology, such as community, hospital-acquired or iatrogenic (Table 3). Gram-positive aerobic organisms, including Streptococcal and Staphylococcus species, account for approximately $65 \%$ of cases were the most frequent organisms identified in community-acquired pleural infection. Meticillin-resistant Staphylococcus Aureus (MRSA) and less commonly cultured Gram-negative organisms, such as E. Coli, Enterobacter spp moreover, Pseudomonas spp which are more common in patients who need admis-
TABLE 3. Bacteriology of the community-acquired and hospital-acquired pleural infection

Common organisms

Community-acquired $\quad$ Streptococcus spp. ( $52 \%)$
S. milleri
S. pneumoniae
S. intermedius
Staphylococcus aureus (11\%)
Gram-negative aerobes (9\%)
Enterobacteriaceae
Escherichia coli
Anaerobes (20\%)
Fusobacterium spp.
Bacteroides spp.
Peptostreptococcus spp.
Mixed
Staphylococci
Methicillin-resistant S. aureus
(MRSA) (25\%)
S. aureus (10\%)
Gram-negative aerobes (17\%)
E. coli
Pseudomonas aeruginosa
Klebsiella spp.
Anaerobes (8\%)

*Adapted from Davies HE, Davies RJ, Davies CW; BTS Pleural Disease Guideline Group. Management of pleural infection in adults: British Thoracic Society Pleural Disease Guideline 2010. Thorax. 2010;65 (Suppl 2): 41-53.

sion to the intensive care unit because of the co-morbidities are responsible for the majority of hospital-acquired pleural infection. The frequency of anaerobic isolates is rising, and positive pleural fluid cultures in most series report anaerobes in $12-34 \%$. However, when identified using different methods, such as DNA amplification, anaerobes may be present in up to $76 \%$ of cases and may be the only pathogen in approximately $14 \%$ of culturepositive cases [2]. In our study, therefore, we have used microorganisms as Klebsiella pneumoniae, Fusobacterium nucleatum and Streptococcus pneumoniae that require more extended and appropriate use of antibiotics. Mixed infection, which is causative bacteria, including gram-positive or negative bacteria, as well as anaerobic bacteria, occurs in many patients with empyema [7]. Parapneumonic effusion and empyema should be administered primarily with intravenous antibiotics. To treat this type of infection, antimicrobial agents are administered for a longer 
period, not less than four weeks from a common respiratory infection, such as pneumonia. When the treatment time is less than two weeks, it is more likely to recur. The initial choice of antibiotics is usually based on whether the patient has community-acquired pneumonia (CAP) or hospital-acquired pneumonia (HAP) and whether the patient is dependent on the seriousness of his or her clinical status. In such cases, especially moxifloxacin is broadly administered because of its broad-range antimicrobial activity against Gram-positive and Gram-negative bacteria, including anaerobes. The penetration of MXF into the pleural fluid is mostly based on experimental pleural empyema in rabbits. Data on penetration in the human pleural fluid are rare. We have found only one publication in the literature. Animal and human results were similar [11]. We wanted to compare the commonly used and proven moxifloxacin with Doripenem.

In pleural infection, pleural effusions can be quickly evolved from uncomplicated parapneumonic effusions to empyema. Classically, empyema is divided into three stages: exudative, fibrin exudation $\&$ pus formation and organization stage [11]. In the exudative period of empyema, pleural fluid accumulates due to the increased permeability of the visceral pleura. As the infection progresses, the accumulation of fibrins on the pleural membranes leads to thick pleura and the formation of septations. Inpatient with empyema, the pleural surfaces are thicker, the pleural fluid is more acidic and more purulent fluid due to inflammation, and increased protein flux is present in the pleural space than with other conditions. It is quite possible antibiotic penetration to be blocked by thickened pleura.

Consequently, in the treatment of microorganisms that cause empyema, the specificity of antibiotics is also essential as the distribution in the body. If the antimicrobial agent does not enter the site at which the offending microorganism resides, bacterial growth will continue despite the in vitro susceptibility of the organism to the drug [6]. Generally, pleural fluids are believed to be similar in serum levels of antibiotics. A fluid balancing between serum and antibiotics depends on many factors. These include the size of the pleural effusion, the thickness of the pleura, the degree of pleural inflammation and the medicine itself. In our study, all factors were the same, except for antibiotics [12]. Doripenem was better than in the penetration rate and equalization time between the $\mathrm{PF}$ and blood. Moxifloxacin is parallel in the amount of blood and pleural fluid. The results of our study suggest that doripenem can be used in suitable patients instead of the empirically used MXF in the treatment of para- pneumonic effusion and empyema. However, this study limitation, as in every study, is that the rabbit pleura thinner than a human, which may increase the thickness of pleural due to empyema. In consequence of pleural fluid penetration of both antibiotics was right, these results derived from animal models can be changed penetration quantity of infected pleural fluid and equilibration between serum, and pleural fluid can be extrapolated to humankind. We believe that doripenem can be used at the treatment of complicated parapneumonic effusion and empyema developed in humankind.

In the literature, there are a lot of experimental studies relating to carbapenems and quinolones. To obtain good clinical results in the empyema, equalization time between the PF and blood and penetration of antibiotics into the infection site is very important. The success of an antimicrobial agent in the treatment of empyema depends on adequate drug concentrations in the pleura, and more specifically, on the achievement of pleural fluid. Thus, the amount and rate of penetration of antibiotics into the pleural space should be kept in mind at initial treatment, and the underlying pathophysiology and the mechanisms of fluid formation should be noticed.

To our knowledge, this is the first study about the pleural penetration of doripenem. No study compared doripenem and moxifloxacin in experimental models of empyema done with these microorganisms. Differences were found in the penetration of the two antibiotics. Doripenem had convenient penetration PF compared to moxifloxacin. Doripenem sufficiently penetrates the pleural space and exhibits an adequate and sufficient pharmacokinetic profile for the treatment of parapneumonic effusion and empyema. Due to the differences between human and rabbit pleural thickness, doripenem's pleural penetration should be examined in infection models in animals with equal pleura thickness and clinical trials.

Ethics Committee Approval: The Selcuk University Clinical Research EthicsCommittee granted aproval for this study (2011-74).

Conflict of Interest: No conflict of interest was declared by the authors.

Financial Disclosure: This study was approved and funded by School of Medicine Animal Care and Investigational Committee in our institution.

Authorship Contributions: Concept - MC, SGC, MD, RK, HE; Design - MC, SGC, MD, RK, HE; Supervision - MC, SGC, MD, RK, HE; Fundings - MC, SGC, MD, RK, HE; Materials - MC, SGC, MD, RK, HE; Data Collection and/or Processing - MC, SGC, MD, RK, HE; Analysis and/or Interpretation - MC, SGC, MD, RK, HE; Literature Review MC, SGC; Writer - MC, SGC; Critical Review - MC, SGC, MD, RK, HE. 


\section{REFERENCES}

1. Rizvi SI, Waller D. Empyema thoracis. Surgery 2011;29:217-20. [CrossRef]

2. Davies HE, Davies RJ, Davies CW; BTS Pleural Disease Guideline Group. Management of pleural infection in adults: British Thoracic Society Pleural Disease Guideline 2010. Thorax 2010;65 Suppl 2:ii41-53.

3. Taylor MD, Kozower BD. Surgical spectrum in the management of empyemas. Thorac Surg Clin 2012;22:431-40. [CrossRef]

4. Saroglou M, Ismailos G, Tryfon S, Liapakis I, Papalois A, Bouros D. Penetration of azithromycin in experimental pleural empyema fluid. Eur J Pharmacol 2010;626:271-5. [CrossRef]

5. Sakran W, Ababseh Zel D, Miron D, Koren A. Thoracic empyema in children: clinical presentation, microbiology analysis and therapeutic options. J Infect Chemother 2014;20:262-5. [CrossRef]

6. Liapakis IE, Kottakis I, Tzatzarakis MN, Tsatsakis AM, Pitiakoudis MS, Ypsilantis P, et al. Penetration of newer quinolones in the empyema fluid. Eur Respir J 2004;24:466-70. [CrossRef]
7. Niwa T, Nakamura A, Kato T, Kutsuna T, Katou K, Morita H, et al. Pharmacokinetic study of pleural fluid penetration of carbapenem antibiotic agents in chemical pleurisy. Respir Med 2006;100:324-31.

8. Chahine EB, Ferrill MJ, Poulakos MN. Doripenem: a new carbapenem antibiotic. Am J Health Syst Pharm 2010;67:2015-24. [CrossRef]

9. AVMA Guidelines for the Euthanasia of Animals: 2013 Edition. Available at: https://www.avma.org/sites/default/files/resources/euthanasia.pdf. Accessed Mar 13, 2020.

10. Yang W, Zhang B, Zhang ZM. Infectious pleural effusion status and treatment progress. J Thorac Dis 2017;9:4690-9. [CrossRef]

11. Chatzika K, Manika K, Kontou P, Pitsiou G, Papakosta D, Zarogoulidis $\mathrm{K}$, et al. Moxifloxacin pharmacokinetics and pleural fluid penetration in patients with pleural effusion. Antimicrob Agents Chemother 2014;58:1315-9. [CrossRef]

12. Teixeira LR, Sasse SA, Villarino MA, Nguyen T, Mulligan ME, Light RW. Antibiotic levels in empyemic pleural fluid. Chest 2000;117:17349. [CrossRef] 